\title{
A STUDY ON MODEL REFERENCE ADAPTIVE PROCESSES OF JAPAN'S REGIONAL DEVELOPMENT IN THE 1970'S
}

\author{
By Etsuo YAMAMURA* and Yuzuru MIYATA**
}

\begin{abstract}
This study aims at considering the trends in the economic development activities within the nine economic regions of Japan from the viewpoint of adaptation process. To achieve this objective, the two approaches below are adopted. Firstly, the potential growth of development activities within Japan in the 1970's is examined by solving the turnpike for the nine regional economies, and then clarifying how the adaptation processes of the actual regional development corresponded to the turnpike. Secondly, the model reference adaptive technique is applied for analyzing warranted regional structural changes which would realize the goal of the New Comprehensive Nationwide Development Plan of Japan.

Keywords: model reference adaptive system, adaptation process, turnpike, regional development, regional planning support system
\end{abstract}

\section{INTRODUCTION}

The purpose of this study is to clarify adaptation processes associated with development within Japan's nine economic regions from 1970 to 1980 , by applying the Model Reference Adaptive Technique which has been developed through our previous studies ${ }^{1) \sim 3)}$.

Our previous studies have aimed at future projection of regional development pattern rather than investigation of past development mechanisms. However, analysis of past development mechanisms, it is thought, would unearth fruitful lessons and suggestions for comparative regional planning and development activities.

In this study, we shall adopt two approaches to realize our stated objectives. The first is to examine the potential growth of development activities within Japan in the 1970's by solving the turnpike for Japan's nine regional economies, and to clarify how the actual regional development adapted to the turnpike.

The second is to analyze warranted regional structural changes which would realize the goal of the New Comprehensive Nationwide Development Plan of Japan. The plan in question is the national land plan initiated by Japan's government in the 1970's. This analysis is done by applying the Model Reference Adaptive Technique.

\section{JAPAN'S NINE REGIONAL TURNPIKE}

In this chapter we shall examine the potential growth and the actual adaptation processes of the Japan's

* Member of JSCE, Dr. Eng., Professor, Department of Environmental Planning, Graduate School of Hokkaido University (Kita 10, Nishi 5, Kita-ku, Sapporo)

** Member of JSCE, M. Env. Sci., Instructor, Department of Environmental Planning, Graduate School of Hokkaido University (Kita 10, Nishi 5, Kita-ku, Sapporo) 
nine regional economies by using the 1970-1980 empirical data.

(1) Dynamic interregional input-output model

The dynamic interregional input-output model (DIRIO) is the main analytical tool for this study. So, we shall offer a brief explanation of it including the definition of notations.

The dynamic balance equation of the Isard type $m$ regional $n$ sectoral interregional input-output system is as follows;

$X(t)=A(t) X(t)+B(t+1) X(t+1)-B(t) X(t)+C S \cdot V(t) X(t)+H$

where, $X(t)=\left(x_{i}^{r}(t)\right)$ : output of sector $i$ in region $r$ at the $t$-th period, $A(t)=\left(a_{i j}^{r s}(t)\right)$ : interregional input coefficient which stands for the input from sector $i$ in region $r$ to sector $j$ in region $s$ at the $t$-th period, $B(t)=\left(b_{i j}^{r s}(t)\right)$ : interregional capital coefficient which stands for the firm's capital investment demand of sector $j$ in region $s$ for the commodity produced by sector $i$ in region $r$ at the $t$-th period, $C S$ : matrix of regional marginal propensities to consume (assumed to be constant), $V(t)$ : regional value added coefficient matrix at the $t$-th period, $H$ : regional basic consumption vector which is independent of income variation (assumed to be constant).

The left hand of (1) stands for the supply in each region and sector while the right hand depicts the demand in each region and sector. The increment of the output from the $t$-th to the $(t+1)$-th period can be determined when the regional outputs, the interregional input coefficients, the interregional capital coefficients, the rates of regional value added and the regional marginal propensities to consume at the $t$-th and/or the $(t+1)$-th period are known. Also (1) can be transformed into the equation below if we assume that the interregional capital coefficient matrix is non-singular i. e. $\operatorname{det} B(t) \neq 0$. The nonsingularity of $B(t)$ means that every sector in each region produces capital goods.

$X(t+1)=B^{-1}(t+1)(I-A(t)-C S \cdot V(t)+B(t)) X(t)-B^{-1}(t+1) H$

(2) The data

On the basis of economic activities, Japan was classified into nine regions. There are Hokkaido, Tohoku, Kanto, Tokai, Hokuriku, Kinki, Chugoku, Shikoku and Kyushu. These areas are depicted in Fig. 1. Japan's interregional input-output tables have been published every five years since 1960. These data served as our main source of data. However, some data had to be estimated, and the main estimation results are presented below.

a) Interregional input coefficient

The interregional input-output tables published by Ministry of International Trade and Industry, Government of Japan (MITI) are constituted by nine regions and ten sectors ${ }^{4)}$. However, the regional classification of 1975 and 1980 Interregional Input-Output Tables were converted into the different classification from the former one. So we unified the regional classification same as in Fig. 1, because Okinawa, which was separated from Kyushu in the 1975's and 1980's tables, was deemed to be too small in our interregional analysis. Furthermore, sectoral divisions were aggregated into one sector for lack of information on interregional sectoral capital flow. These rearrangements were made by applying Annual Report of Prefectural Accounts ${ }^{5}$. The estimation results are explained briefly as follows.

The regional value added rates from 1970 to 1980 , as depicted in Fig. 2, tended to decrease gently rather than a uniform increase, the reason, perhaps, being that the share rate of the manufacturing industry expanded. The manufacturing industry has a relatively high technological progress but its value added rate is lower than the tertiary industry,

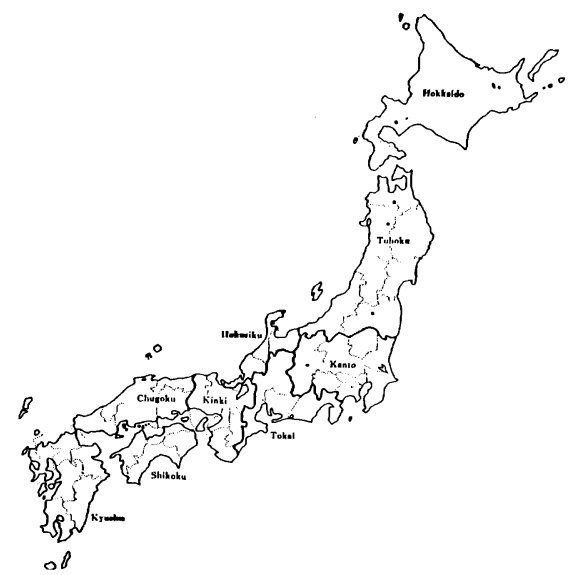

Fig. 1 Classification of Nine Regions in Japan. 


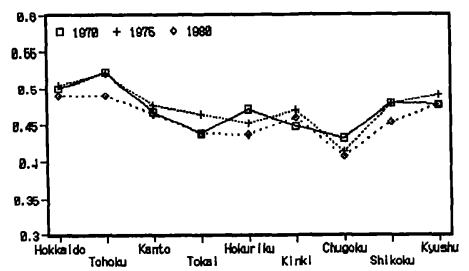

Fig. 2 Actual Regional Value Added Rates.

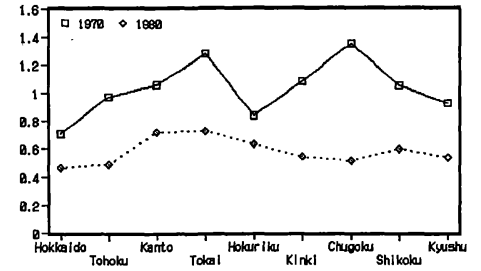

Fig. 3 Actual Regional Capital Productivities.

which represented more than $50 \sim 65 \%$ share rate in each region ${ }^{5)}$. Tohoku, Hokkaido, and Kyushu are portrayed as the regions with high value added. This fact shows a specific Japan's regional characteristic, that is, local regions tend to have higher value added than Kanto, Kinki and Tokai in which the major metropolitan areas such as Tokyo, Osaka and Nagoya are located. And the value added rate of Chugoku is lowest among the nine regions.

b) Interregional capital coefficient

The level of capital stock used in this study were derived from stocks owned by both public and private firms. Though inventory is usually regarded as a part of capital stock, we considered the net increment of inventory as consumption because of instability of the inventory data.

The estimation of the interregional capital stock matrices was done by employing the next equation.

$$
k_{r s}(t)=\left(\sum_{m=t-5}^{t} I_{r s}(m)\right) /\left(x_{s}(t+1)-x_{s}(t-5)\right) \quad(t=1970,1975,1980)
$$

where, $k_{r s}(t)$ : capital stock owned in region $s$ produced by region $r, I_{r s}(t)$ : capital formation from region $r$ to region $s, x_{s}(t)$ : output of region $s$.

The reason why the capital investments are accumulated in these equations is to eliminate bias caused by business fluctuations. The interregional capital coefficients were calculated by

$$
b_{r s}(t)=k_{r s}(t) / x_{s}(t) \quad(t=1970,1975,1980)
$$

But we did not obtain good estimation results for 1975's matrix as larger fluctuations were obtained for some regions. Thus we would like to leave the 1975 's capital coefficient matrix as a reference.

The regional capital productivities in 1970 and 1980 are presented in Fig. 3. Comparing these regional capital productivities, we observe that those of 1980 are smaller in relation to their corresponding figures for 1970 in all regions. This means that the regional capital stocks have accumulated, however, the regional capital productivities did not get higher with the regional capital accumulation. From a regional point of view, the regional capital productivity is highest in Chugoku, then Tokai, Kinki, Kanto, Shikoku, Tohoku, Kyushu, Hokuriku and Hokkaido followed in that order in 1970. It is pointed out that the capital productivities of the regions where heavy industries were located were chiefly higher. In 1980, the regional capital productivities followed in the order that Tokai, Kanto, Hokuriku, Shikoku, Kinki, Kyushu, Chugoku, Tohoku and Hokkaido. Chugoku, Kinki, Tohoku, Tokai, Shikoku, and Kyushu decreased more than $40 \%$, and this suggests that there were over-equipped capital stocks in heavy industries.

( 3 ) Regional turnpike of Japan's nine regional economies from 1970 to 1980

Based on the above data, the calculation of the regional turnpike for the nine regions is as follows. Capital accumulation turnpike is given by a solution of the next programming problem ${ }^{6}$.

approximation programming of regional turnpike (APRT)

$\max \alpha$

subject to $(I-A(t)-C S \cdot V(t)+B(t)) X(t)-H \geqq B(t+1) X(t+1) \quad(t=0,1, \cdots, T-2)$

$(I-A(T-1)-C S \cdot V(T-1)+B(T-1)) X(T-1)-H$

$\geqq B(T)\left(\alpha \mu(T)+(I-A(T-1)-C S \cdot V(T-1))^{-1} H\right) \cdot$

$$
X(t) \geqq 0 \quad(t=0,1, \cdots, T-1)
$$


where, $T:$ planned period, $\mu(T):$ an eigen vector corresponding to $1+1 / \lambda(T), \lambda(T):$ Frobenius root of $(I-A(T-1)-C S \cdot V(T-1)-B(T-1)+B(T))^{-1} B(T)$.

This programming technique ensures that regional growth path approaches the turnpike at period $T$. The previous studies have shown that as APRT is solved, it approaches the turnpike in the initial periods. But as it is well known, turnpike trajectory takes a downturn at the second period, and then increases along a balanced growth path. This decreasing tendency has been pointed out as unrealistic by many economists. So Tsukui, J. introduced in his book ${ }^{7)}$ additional activities, that is, temporal stock activities and temporal trade activities so that the turnpike programming technique could be more realistic. The temporal stock activities transfer the unused stock of goods in some period to the next period without any cost. The temporal trade activities involve the export of unused stock of goods and the import of urgently needed commodities. These activities are denoted as $S(t)=\left(s_{r}(t)\right)$ and $E(t)=\left(e_{r}(t)\right)$ respectively, here. So we can construct another programming technique named the programming of regional turnpike with adjustment activities, which is defined as follows;

programming of regional turnpike with adjustment activities (PRTAA)

$\max a$

subject to $(I-A(t)-C S \cdot V(t)+B(t)) X(t)-H+E(t)+S(t) \geqq B(t+1) X(t+1)+S(t+1)$

$(t=0,1, \cdots, T-2)$.

$(I-A(T-1)-C S \cdot V(T-1)+B(T-1)) X(T-1)-H+E(T-1)+S(T-1)$

$\geqq B(T)\left(\alpha \mu(T)+(I-A(T-1)-C S \cdot V(T-1))^{-1} H\right)+S(T)$

$\sum_{r=1}^{9} e_{r}(t)=0 \quad(t=0,1, \cdots, T-1)$

$$
X(t) \geqq 0 \quad(t=0,1, \cdots, T-1)
$$

This programming ensures that regional outputs approach the turnpike more smoothly and realistically.

Then the eigen values and the associated eigen vectors of $(I-A(t)-C S \cdot V(t)+B(t)-B(t+1))^{-1}$ $B(t+1)$ from 1970 to 1980 were solved by the power method. Because each $(I-A(t)-C S \cdot V(t)+B(t)-$ $B(t+1))^{-1} B(t+1)$ is positive and indecomposable, $(I-A(t)-C S \cdot V(t)+B(t)-B(t+1))^{-1} B(t+1)$ has a positive eigen value with the maximum absolute value among the eigen values of $(I-A(t)-C S \cdot V(t)+B(t)$ $-B(t+1))^{-1} B(t+1)$ by the Frobenius Theorem. And the value $1 / \lambda(t+1)(\lambda(t+1)$ which is the Frobenius root of $\left.(I-A(t)-C S \cdot V(t)+B(t)-B(t+1))^{-1} B(t+1)\right)$ stands for the annual growth rate of the balanced growth path from the $t$-th to the $(t+1)$-th period. The computation results of $1 / \lambda(t+1)$ are presented in Fig. 4 . We observe that the balanced growth rate decreases gradually from 1971 to 1980 . The reason is that the capital-output ratio of the regions increase from 1970 to 1980, therefore by the acceleration principle, annual increment of regional output needed to induce regional private investment may get smaller.

Next, we shall refer to the eigen values and their associated eigen vectors. The eigen vector corresponding to the Frobenius root stands

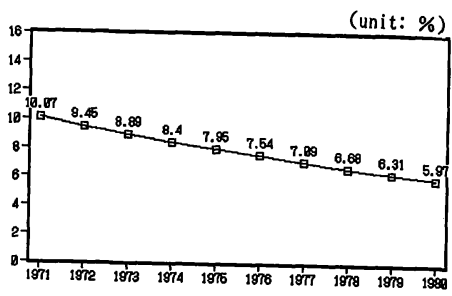

Fig. 4 Trend of Balanced Growth Rate. for the optimal interregional industrial location rate. Fig. $5 \sim 7$ illustrate the comparison of the optimal industrial location rate and the actual share rate of the regional outputs of 1970, 1975 and 1980. Kanto's actual share rate exceeds the optimal one from 1970 through 1980, and this means that the over-concentration of industrial activities in Kanto stands out among the other regions. On the other hand, the actual share rates of Kinki and Chugoku are lower than the optimal rate. These facts show an existence of imbalance of the interregional industrial location. Concerning Hokkaido, Tohoku, Tokai, Hokuriku, Shikoku and Kyushu, their actual share rates depict effective location rates, by reason of the few differences existing between the actual share rate and the corresponding optimal rate.

The regional turnpike from 1970 to 1980 calculated by PRTAA are presented in Fig. $8 \sim 10$. These 


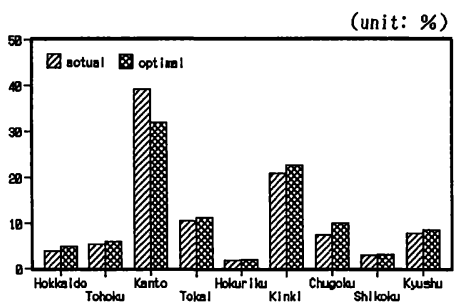

Fig. 5 Actual and Optimal Share Rates of Regional Outputs in 1970 .

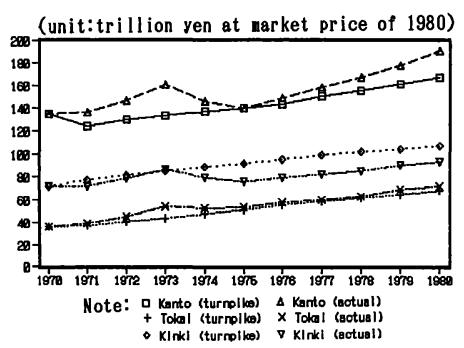

Fig. 8 Regional Turnpike and Actual Outputs.

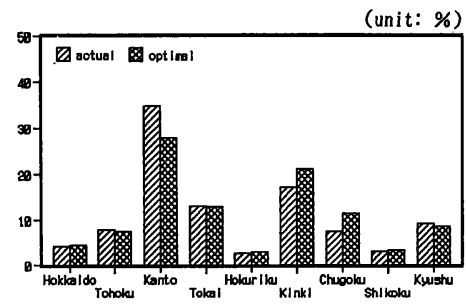

Fig. 6 Actual and Optimal Share Rates of Regional Outputs in 1975.

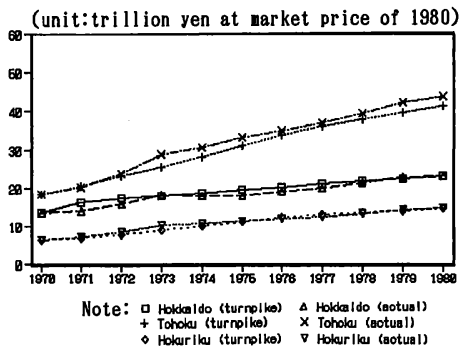

Fig. 9 Regional Turnpike and Actual Outputs.

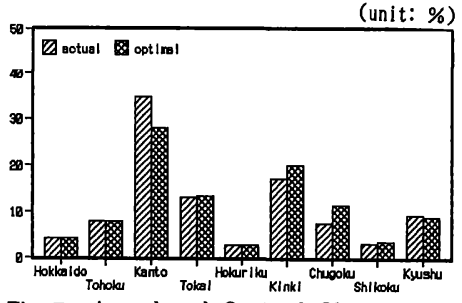

Fig. 7 Actual and Optimal Share Rates of Regional Outputs in 1980 .

regional turnpike paths grow at variable rates annually, because of the variation of the actual interregional input and capital coefficients. The highest annual average growth rate is that of Hokuriku, $8.43 \%$. The estimated growth rates for the other regions are as follows; Tohoku $8.40 \%$, Chugoku $7.82 \%$, Tokai $6.43 \%$, Shikoku 6.04\%, Hokkaido 5. $40 \%$, Kinki $4.03 \%$, Kyushu 2. $48 \%$ and Kanto $2.19 \%$. Comparing these turnpike paths to the actual regional outputs from 1970 to 1980, it is observed that Chugoku's outputs are exceedingly lower than its turnpike outputs after 1974, while Kanto's outputs are much larger than the turnpike path from 1975.

Now, let us introduce here a new index, a degree of adaptation, in order to measure the differences between the turnpike outputs and the actual ones.

degree of adaptation

$$
D A(t)=\sum_{i=1}^{n}\left|x_{i}^{*}(t)-x_{i}(t)\right| / x_{i}^{*}(t) \times 100
$$

where, $x_{i}^{*}(t):$ turnpike output of region $i$ at the $t$-th period

$x_{i}(t)$ : actual output of region $i$ at the $t$-th period

$D A(t)$ gets nearer to zero as the actual outputs approach the turnpike paths, and it increases more and more as the actual outputs deviate from the turnpike trajectories. Fig. 11 shows the trends of the degree of adaptation with respect to both the nine regions $D A_{9}(t)$ and thereafter, the seven regions $D A_{7}(t)$ (excluding Kanto and Hokuriku) from 1970 to 1980 . $D A_{9}(t)$ shows a slight increase from 1974 and assumes a level after 1977 . Therefore all of the actual regional outputs seem not to approach the turnpike trajectories. On the other

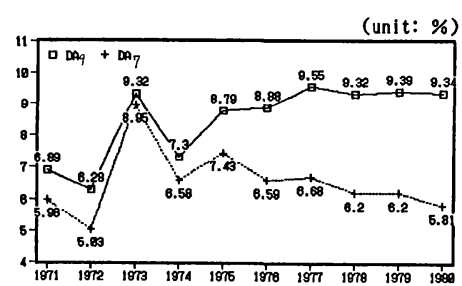

Fig. 11 Degree of Adaptation to Regional Turnpike. hand, as $D A_{7}(t)$ tends to approach zero after 1975, the actual outputs except Kanto and Chugoku are interpreted to be gradually approaching the turnpike.

As mentioned above, the Japan's nine regional development processes from 1970 to 1980 can be characterized by the large concentration of industries in Kanto, the stagnation of growth within Chugoku and the adaptation of the remaining regional outputs to the regional turnpike. 


\section{MODEL REFERENCE ADAPTIVE PROCESSES IN JAPAN'S NINE REGIONAL ECONOMIES}

The previouce chapter showed the development trends within the nine economic regions of Japan between 1970 and 1980 from the viewpoint of the adaptation to the regional turnpike. In this chapter, we shall consider how adaptation would be needed to ensure a more desirable state of development within these regions. For this objective, we have to establish a more desirable state of the Japan's regional development. So we would like to consider a more desirable state of the regional development based on the past Japan's nationwide development plan rather than the conventional optimization type models. The plan introduced hereafter is the New Comprehensive Nationwide Development Plan, a plan which was initiated by the government and centered on national land development schemes from 1970 to 1980 .

(1) The new comprehensive nationwide development plan

In 1962 the first national land plan of Japan, that is, the Comprehensive Nationwide Development Plan (CNDP) was instituted ${ }^{8}$. Under this plan, the Program for the Promotion of Strategic Regions was proposed in order to resolve the depopulation and overpopulation problems, which had been a major nationwide development obstacle at that time, and to realize more effective national land development.

Even though Japan had achieved the higher economic growth without parallel in the world during the 1960's, overpopulation evils in the major urbanized area had left much to be desired. These diseconomies triggered off the need for a new national land plan, resulting in the institution of the New Comprehensive Nationwide Development Plan (NCNDP) in 1969). The targets of the NCNDP were to cope with information oriented, internationalized, and highly technological society that was evolving in each region as well as solving the depopulation and overpopulation problem which had been the major thrust of the CNDP. And the Big Project Program was introduced to put into effect and realize the aims of the NCNDP. The Big Project Program aimed at the arrangement of nationwide transportation and telecommunication networks, which was expected to be a fundamental infrastructure for the nationwide develop-

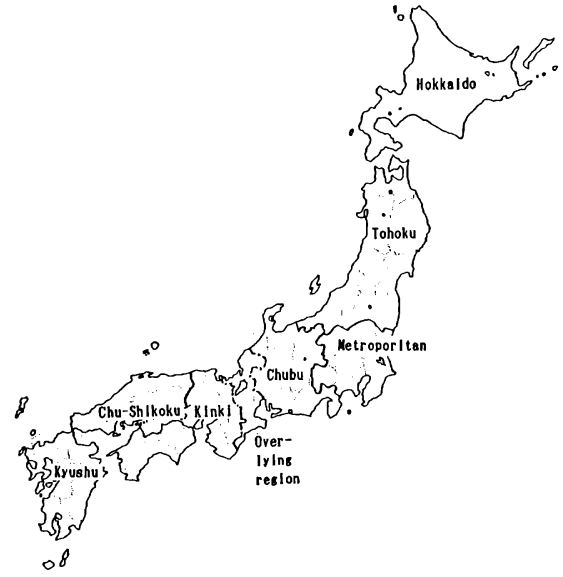

Fig. 12 Regional Classification of Japan in the NCNDP. ment, for systematizing the cluster of central administrative function and organization of physical distribution. In addition, this programm aimed at the planning and implementation of independent and effective large projects for industrial development and environmental conservation, utilizing the networks and regional resources in order to initiate balanced nationwide land use as well as to develop each region.

The effect of Big Project Program was forecasted as a dispersed national land development as presented in Table 19). Case 1 shows that the trend of the regional growth during the 1950's and the 1960's would continue, while case 2 represents one with a precondition that the Big Project Program would work out well.

\section{( 2 ) Model reference adaptive processes in Japan's nine regional development}

The regional turnpike presented in Chapter 2 was calculated to reflect the influence of social or regional problem such as the oil shock and public nuisance in the 1970's. So we shall consider in this section how regional structural changes could realize the more desirable state of the nationwide regional development when the frame of the NCNDP is assumed to be more desirable. The approach of MRAS, which has been developed through our previous studies, may offer some useful insights looking at this problem. As mentioned in the previous paper, we explain the reference and the adaptive models in this order. Firstly, 
Table 1 Frame of the New Comprehensive Nationwide Development Plan.

\begin{tabular}{|c|c|c|c|c|c|c|c|c|c|c|}
\hline \multirow[b]{2}{*}{ resion } & \multicolumn{2}{|c|}{1965 (actual) } & \multicolumn{2}{|c|}{ 1985 (case 1) } & \multicolumn{2}{|c|}{ 1985(case 2) } & \multicolumn{2}{|c|}{ gr.rate(casel) } & \multicolumn{2}{|c|}{ sr.rate(case2) } \\
\hline & pop. & product & pop. & product & pop. & product & pop. & prod: & pop. & prod. \\
\hline Hokka ido & $\begin{array}{c}517 \\
(5.10)\end{array}$ & $\begin{array}{l}1,134 \\
(4.45)\end{array}$ & $\begin{array}{c}470 \\
\text { (3.78) }\end{array}$ & $\begin{array}{l}4,400 \\
(3.39)\end{array}$ & $\begin{array}{c}630 \\
(5.09)\end{array}$ & $\begin{array}{l}5,800 \\
(4.51)\end{array}$ & -0.48 & 7.01 & 0.99 & 8.50 \\
\hline Tohoku & $\begin{array}{c}1.151 \\
\text { (ii.35) }\end{array}$ & $\begin{array}{l}2.236 \\
(8.78)\end{array}$ & $\begin{array}{c}970 \\
(7.81)\end{array}$ & $\begin{array}{l}7,700 \\
(5.93)\end{array}$ & $\begin{array}{l}1,100 \\
(8.89)\end{array}$ & $\begin{array}{l}9,600 \\
(7.47)\end{array}$ & -0.85 & 6.38 & -0.23 & 7.56 \\
\hline Metropolitan & $\begin{array}{c}2,696 \\
(26.59)\end{array}$ & $\begin{array}{c}8.002 \\
(31.43)\end{array}$ & $\begin{array}{c}4.050 \\
(32.61)\end{array}$ & $\begin{array}{l}50.000 \\
(38.49)\end{array}$ & $\begin{array}{r}3,850 \\
(31.10)\end{array}$ & $\begin{array}{l}42,800 \\
(33.31)\end{array}$ & 2.06 & 9.59 & 1.80 & 8.75 \\
\hline Chubu & $\begin{array}{c}1,649 \\
(16.26)\end{array}$ & $\begin{array}{c}4.002 \\
(15.72)\end{array}$ & $\begin{array}{c}2.250 \\
(18.13)\end{array}$ & $\begin{array}{l}23.000 \\
(17.71)\end{array}$ & $\begin{array}{r}2,050 \\
(16.56)\end{array}$ & $\begin{array}{l}22,000 \\
(17,12)\end{array}$ & 1.57 & 9.14 & 1.09 & 8.89 \\
\hline Kink & $\begin{array}{l}1,804 \\
(17.79)\end{array}$ & $\begin{array}{c}5.282 \\
(20.75)\end{array}$ & $\begin{array}{c}2,600 \\
(20.93)\end{array}$ & $\begin{array}{l}28,500 \\
(21.94)\end{array}$ & $\begin{array}{c}2,450 \\
(19.79)\end{array}$ & $\begin{array}{l}26,600 \\
(20.70)\end{array}$ & 1.84 & 8.79 & 1.54 & 8.42 \\
\hline Chu-Shikok & $\begin{array}{c}1,085 \\
(10.70)\end{array}$ & $\begin{array}{c}2.423 \\
(9.52)\end{array}$ & $\begin{array}{r}980 \\
(7.89)\end{array}$ & $\begin{array}{l}8,300 \\
(6.39)\end{array}$ & $\begin{array}{l}1,100 \\
(8.89)\end{array}$ & $\begin{array}{c}10,800 \\
(8.40)\end{array}$ & -0.51 & 6.35 & 0.07 & $7.7 \mathrm{~B}$ \\
\hline Kyushu & $\begin{array}{l}1,237 \\
(12.20)\end{array}$ & $\begin{array}{l}2,382 \\
(9.36)\end{array}$ & $\begin{array}{l}1.100 \\
(8.86)\end{array}$ & $\begin{array}{l}8.000 \\
(6.16)\end{array}$ & $\begin{array}{l}1.200 \\
(9.69)\end{array}$ & $\begin{array}{c}10,900 \\
(8.48)\end{array}$ & -0.59 & B.24 & -0.15 & 7.90 \\
\hline Total & $\begin{array}{l}10,139 \\
(100.00)\end{array}$ & $\begin{array}{c}25.461 \\
(100.00)\end{array}$ & $\begin{array}{l}12,420 \\
(100.00)\end{array}$ & $\begin{array}{l}129,900 \\
(100.00)\end{array}$ & $\begin{array}{r}12,380 \\
(100.00)\end{array}$ & $\begin{array}{l}128.500 \\
(100.00)\end{array}$ & 1.02 & 8.49 & 1.00 & 8.43 \\
\hline
\end{tabular}

Note 1: (unit) population in ten thousand persons, product in billion yen at earket price of 1965. share and growth rates in $\%$ Note 2: share rates are in parantheses.
Table 2 Frame of Outputs for the NCNDP.

\begin{tabular}{|c|c|c|c|}
\hline resion & 1970 & 1980 & gr. rate \\
\hline Hokka Ido & & & 8.01 \\
\hline rohoku & 18,444 & $\begin{array}{r}(4.61) \\
55,725\end{array}$ & 11.69 \\
\hline Kanto & $\begin{array}{r}(5.36) \\
134.708\end{array}$ & $\begin{array}{r}(7.98) \\
247.499\end{array}$ & 8.27 \\
\hline & $(39.16)$ & $(35.43)$ & \\
\hline Tokal & 36 & 83,065 & 8.84 \\
\hline Hokur Iku & 2 & $\begin{array}{l}18.057 \\
18.097\end{array}$ & 10.82 \\
\hline & & & 8, \\
\hline & $(20.84)$ & (19.10) & 8.41 \\
\hline Chus oku & 25,604 & 41.428 & 4.93 \\
\hline Shlkoku & 8 & 25. & 7.20 \\
\hline & $(3.00)$ & $(2.98)$ & \\
\hline Ryushu & $\begin{array}{l}26.935 \\
(7.83)\end{array}$ & $\begin{array}{l}86.520 \\
(9.52)\end{array}$ & 9.48 \\
\hline Total & $\begin{array}{r}344.017 \\
(100.00)\end{array}$ & $\begin{array}{l}698.803 \\
(100.00)\end{array}$ & 7.34 \\
\hline
\end{tabular}

let us derive the reference regional outputs because it is enough to give only the information of reference outputs for applying MRAS theory even if the parameters of the reference model are unknown. We set the reference regional outputs as presented in Table 2 with reference to the dispersed state of the NCNDP. The estimation of Table 2 were made by applying Table 1, the Japan's prefectural accounts and interregional input-output tables.

Now, the reference and the adaptive models are defined as follows; reference model

$$
X m(t+1)=f(X m(t), H)
$$

adaptive model

$$
X(t+1)=B^{-1}(t+1)(I-A(t)-C S \cdot V(t)+B(t)) X(t)-B^{-1}(t+1) H
$$

where, $X m(t)$ : reference regional output vector exogenously derived from Table 2. The outputs from 1971 through 1979 were estimated by using the growth rates from 1970 to 1980 which is presented in Table 2, $X(t)$ : adaptive regional output vector, $A(t)$ : adaptive interregional input coefficient matrix, $B(t)$ : adaptive interregional capital coefficient matrix, $C S$ : matrix of adaptive regional marginal propensity to consume, $V(T)$ : adaptive regional value added coefficient matrix.

Each initial value of the above variables is set as the actual value of 1970 . And due to the theorem mentioned in the previous paper ${ }^{1)}$, we can make $\lim _{t \rightarrow \infty}\|\varepsilon(t)\|=\lim _{t \rightarrow \infty}\|X m(t)-X(t)\|=0$ by applying the below-stated adaptation laws.

$$
\begin{aligned}
& C(t+1)=C(t)+(I+\Gamma(t))^{-1} K c \otimes \hat{\varepsilon}(t+1) X^{T}(t) \\
& D(t+1)=D(t)+(I+\Gamma(t))^{-1} K d \otimes \hat{\varepsilon}(t+1) H^{T} \ldots .
\end{aligned}
$$

where,

$$
\begin{aligned}
& C(t+1)=B^{-1}(t+1)(I-A(t)-C S \cdot V(t)+B(t)) \cdots \\
& D(t+1)=-B(t+1)^{-1} \\
& \hat{\varepsilon}(t+1)=X m(t+1)-C(t) X(t)-D(t) H \\
& K c=\left(k c_{i j}\right), K d=\left(k d_{i j}\right) \quad k c_{i j}, k d_{i j}>0 \text {. } \\
& \Gamma(t)=\left[\begin{array}{ccc}
\sum_{s=1}^{9}\left\{k c_{1 s} x_{s}^{2}(t)+k d_{1 s} h_{s}^{2}(t)\right\} & 0 \\
0 & \ddots & \\
& \sum_{s=1}^{9}\left\{k c_{9 s} x_{s}^{2}(t)+k d_{9_{s}} h_{s}^{2}(t)\right\}
\end{array}\right] .
\end{aligned}
$$

$\otimes$ stands for the next matrix operation.

$$
\left[\begin{array}{ccc}
a_{11} & \cdots & a_{1 n} \\
\vdots & & \vdots \\
a_{n 1} & \cdots & a_{n n}
\end{array}\right] \otimes\left[\begin{array}{ccc}
b_{11} & \cdots & b_{1 n} \\
\vdots & & \vdots \\
b_{n 1} & \cdots & b_{n n}
\end{array}\right]=\left[\begin{array}{ccc}
a_{11} b_{11} & \cdots & a_{1 n} b_{1 n} \\
\vdots & & \vdots \\
a_{n 1} b_{n 1} & \cdots & a_{n n} b_{n n}
\end{array}\right]
$$

$A(t), B(t)$ and $V(t)$ are solved by using $C(t+1)$ and $D(t+1)$ as follows; 


$$
\begin{aligned}
& a_{r s}(t)=(I-B(t)-B(t+1) C(t+1))_{r s} \quad(r \neq s) \\
& a_{s s}(t)=\left\{(I-B(t)-B(t+1) C(t+1))_{s s}-c s_{s}+\sum_{r=s} a_{r s}(t)\right\} /\left(1-c s_{s}\right) \\
& \cdot B(t+1)=-D^{-1}(t+1) \\
& v_{s}(t)=1-\sum_{r=1}^{9} a_{r s}(t)
\end{aligned}
$$

Though regional structural changes for realizing the target output paths depicted in Table 2 can be solved theoretically by applying the above-mentioned adaptation laws, some problems are encountered in this computation. Firstly, each adaptive behaviour of $C(t+1)$ and $D(t+1)$ depends on $X(t)$ and $H$, respectively. But $H$ is assumed to be a constant vector in this study, and its values are slightly smaller than $X(t)$. Therefore $C(t+1)$ would fluctuate larger than $D(t+1)$. This leads to the fact that $A(t)$ would experience a bigger change as compared to $B(t)$ due to $(25) \sim(27)$. But as Fig. 2 and 3 show, the behaviour of $A(t)$ is much more stable than $B(t)$. Thus the large fluctuation of $A(t)$ is simply unrealistic. Secondly, it would be expected that the Solow's condition of column summation and/or nonnegativity condition with respect to $A(t)$ would not be ensured if $K c$ or $K d$ is not suitable, because of larger changes of $A(t)$. Accordingly, we shall set two other cases in addition to the ordinary adaptation process.

(3) The simulation results

\section{a) Case 1}

Case 1 is a simulation of simultaneous adaptation of both the interregional input and capital coefficients, and this is the same as one which we have illustrated in our previous papers. The reference and the adaptive models, and the adaptation laws are set up as follows;

reference model

$$
X m(t+1)=f(X m(t), H)
$$

adaptive model

$$
X(t+1)=B^{-1}(t+1)(I-A(t)-C S \cdot V(t)+B(t)) X(t)-B^{-1}(t+1) H \cdot
$$

adaptation laws

$$
\begin{aligned}
& C(t+1)=C(t)+(I+\Gamma(t))^{-1} K c \otimes \hat{\varepsilon}(t+1) X^{T}(t) \\
& D(t+1)=D(t)+(I+\Gamma(t))^{-1} K d \otimes \hat{\varepsilon}(t+1) H^{T} \ldots \\
& \hat{\varepsilon}(t+1)=X m(t+1)-C(t) X(t)-D(t) H \cdots \cdots \ldots . .
\end{aligned}
$$

The notations are the same as those in the previous section, and $K c$ and $K d$ are set up as $k c_{i j}=10^{-8}$ and $k d_{i j}=1 /\left\{x m_{i}(1970) \cdot h_{j}(1970)\right\}$, respectively.

The simulation results of the adaptive regional outputs are evaluated by the degree of adaptation $D A(t)$, which is illustrated in Fig. 13. Despite the fact that the error between the adaptive and the reference outputs is $0.25 \%$ in 1971 , the errors during $1972 \sim 1976$ are less than $0.01 \%$ and also less than $0.015 \%$ from 1977 to 1980. The adaptive model shows a close correspondence to the NCNDP frame. Next, we shall analyze the adaptive behaviour of the interregional technological system by means of the regional input and capital coefficients,

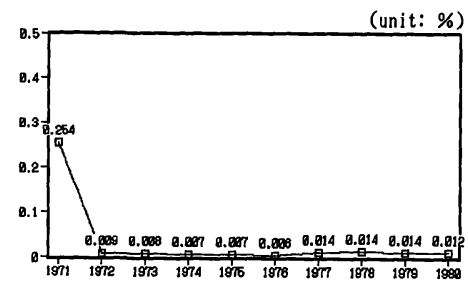

Fig. 13 Degree of Adaptation (case 1)

$$
a_{s}(t)=\sum_{r=1}^{9} a_{r s}(t), b_{s}(t)=\sum_{r=1}^{9} b_{r s}(t)
$$

the reason being that the simulation results of the adaptive interregional input and capital coefficients are too massive to present in this article.

As for the regional input coefficients, Fig. 14 19 denote an increase for all the coefficients. These results mean that the regional value added rate decreases in all regions, but this seems a little unrealistic. The reason is attributed to little fluctuations of the regional capital coefficients as presented in Fig. 17 


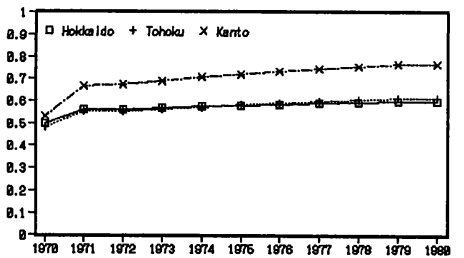

Fig. 14 Adaptive Regional Input Coefficients (case 1).

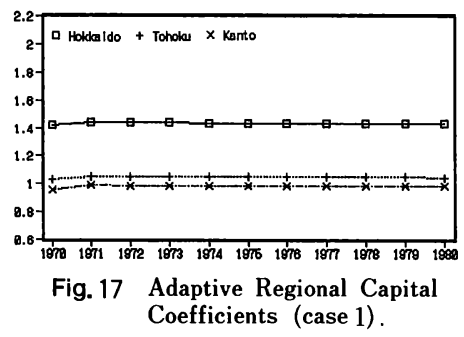

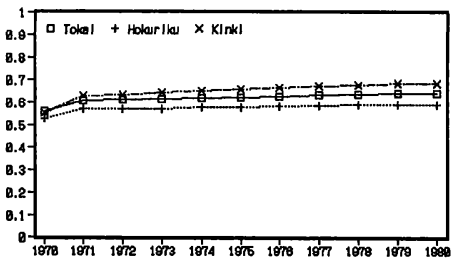

Fig. 15 Adaptive Regional Input Coefficients (case 1).

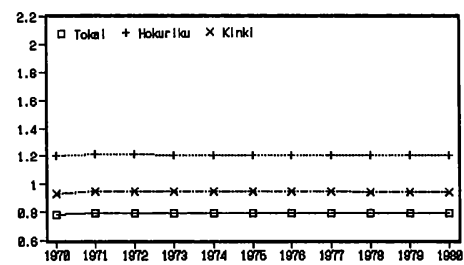

Fig. 18 Adaptive Regional Capital Coefficients (case 1).

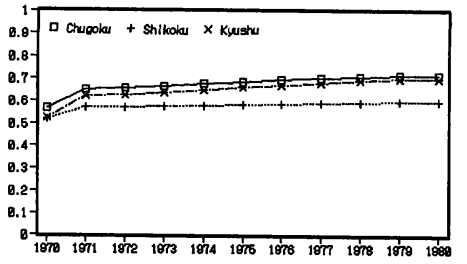

Fig. 16 Adaptive Regional Input Coefficients (case 1).

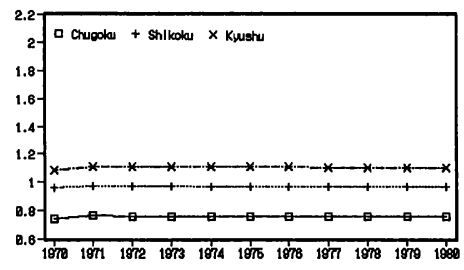

Fig. 19 Adaptive Regional Capital Coefficients (case 1).

19. Now, let us reconsider a balance equation of the dynamic I-O model again.

$$
X(t)=A(t) X(t)+C S \cdot V(t)+B(t+1) X(t+1)-B(t) X(t)+H \cdot
$$

As (35) stands for a static balanced state, for example, if $A(t) X(t)$ is getting larger, the other term must be getting smaller. When the regional capital coefficients do not vary largely, case 1 being so, the induced private investment $B(t+1) X(t+1)-B(t) X(t)$ is regarded as the smaller one. Therefore, large variations of $A(t) X(t)$ are needed to realize a big growth such as one demanded in the NCNDP, and those variations lead to larger interregional input coefficients $A(t)$. The reasons for little variations in $B(t)$ seem to be that the regional basic consumption $H$ is much smaller as compared to the regional outputs $X(t)$ and also the presence of a constant vector.

Besides this, we also attempted some further simulations with other $K c$ and $K d$, which determine the adaptation speed and pattern of adaptive outputs. This is necessary because of the unrealistic regional structural changes obtained in this case. But all the simulation results showed that the adaptive interregional capital coefficients varied slowly with large changes in the adaptive interregional input coefficients. Thus, we could not obtain an adaptive regional structural change that was acceptable, and perhaps realize the actual situation. We would like to leave this problem as a future task. A possibility for solving this problem might be by transforming $K c$ and $K d$ into time variables.

b) Case 2

Case 2 aims at simulating adaptive behaviour of the interregional input coefficients $A(t)$ under the assumption that the interregional capital coefficient matrices $B(t)$ represent the actual ones from 1970 to 1980. The reference and the adaptive models, and the adaptation law are given as follows; reference model

$$
X m(t+1)=f(X m(t), H)
$$

adaptive model

$$
X(t+1)=B m^{-1}(t+1)(I-A(t)-C S \cdot V(t)+B m(t)) X(t)-B m^{-1}(t+1) H
$$

adaptation laws

$$
\begin{aligned}
& C(t+1)=C(t)+(I+\Gamma(t))^{-1} K c \otimes \hat{\varepsilon}(t+1) X^{T}(t) \\
& D(t+1)=-B m^{-1}(t+1) \cdots \cdots \cdots \cdots \cdots \ldots \ldots \ldots \ldots \ldots \ldots \ldots \ldots \ldots
\end{aligned}
$$

$A(t)$ is solved as follows;

$$
\begin{aligned}
& a_{r s}(t)=(I-B m(t)-B m(t+1) C(t+1))_{r s} \quad(r \neq s) \ldots \ldots \ldots \ldots \ldots \ldots \ldots \ldots \ldots \ldots \ldots \ldots \ldots \\
& a_{s s}(t)=\left\{(I-B m(t)-B m(t+1) C(t+1))_{s s}-c s_{s}+\sum_{r=s} a_{r s}(t)\right\} /\left(1-c s_{s}\right)
\end{aligned}
$$




$$
v_{s}(t)=1-\sum_{r=1}^{9} a_{r s}(t)
$$

The simulation was done with $k c_{i j}=1 /\left\{x m_{i}(1970) \cdot x m_{j}(1970)\right\}$. The degree of adaptation of the adaptive outputs to the reference one is depicted in Fig. 20. Because only $A(t)$ is adjusted in case 2, the degree of adaptation is worse than the one in case 1 with more than $6 \%$ error rates for 1971 and 1972. $D A(t)$ decreases uniformly after 1973, but again increases slightly in 1979 and 1980. However, the adaptive regional outputs seem to approach the frame of the NCNDP considerably, because $D A(t)$ shows $1.26 \%$ error rate in 1980 .

The adaptive variations of the interregional input coefficients, which realize the trend of the adaptive regional outputs, are illustrated in Fig. $21 \sim 23$. The adaptive regional input coefficients decrease largely in many regions from 1970 to 1972 or 1973, and assume an increase, then decrease again. Also, the adaptive regional input coefficients, excepting Kinki, get smaller than the initial values, that is to say, there is an adaptation process of $A(t)$ with the regional value added getting higher. The reason for these variations is that the actual interregional capital coefficients are larger than the one which is latently assumed in the NCNDP, in other words, the regional capital productivities are relatively lower. To be more explicit, the actual regional capital productivities from 1970 to 1980 were too low to realize the higher growth postulated in the NCNDP, therefore technological progress to make the intermediate input decrease was needed. As for Hokuriku, its adaptive input coefficient increases largely from 1972 to 1977, despite the fact that its capital coefficient does not seem to be so small. This notion may be attributed to the fact that the decrease of the regional input coefficient from 1970 to 1972 is too large and adjustment after 1972 to counter that decrease is also equally large.

As mentioned above, the adaptive behaviour of the regional input coefficients, in particular in Tohoku, Hokuriku, Kinki and Chugoku, has proved to be unrealistic. Therefore, we can say that the realization of the higher growth oriented and dispersed society embodied in the NCNDP was very difficult under the assumption of the actual interregional capital flow.

c) Case 3

Adaptive changes of the interregional capital coefficients $B(t)$ are simulated in case 3 assuming the interregional input coefficients $A(t)$ in the adaptive model to be the actual ones from 1970 to 1980 . Similar to cases 1 and 2, the reference and the adaptive models, and the adaptation law are set as follows;

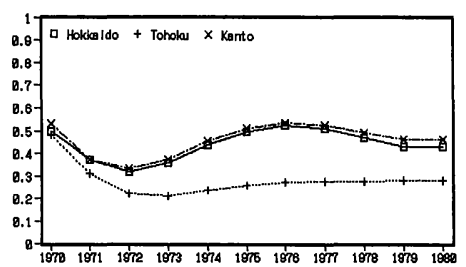

Fig. 21 Adaptive Regional Input Coefficients (case 2).

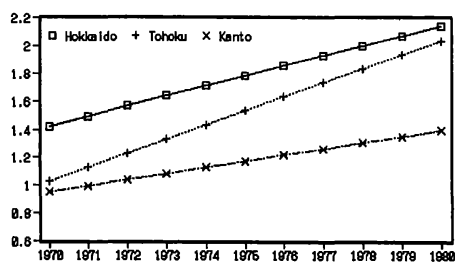

Fig. 24 Adaptive Regional Capital Coefficients (case 2).

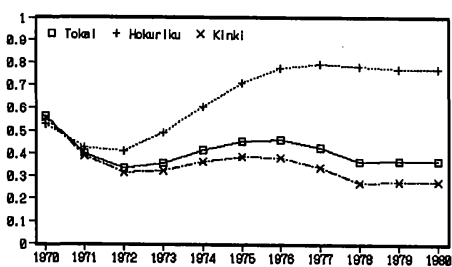

Fig. 22 Adaptive Regional Input Coefficients (case 2).

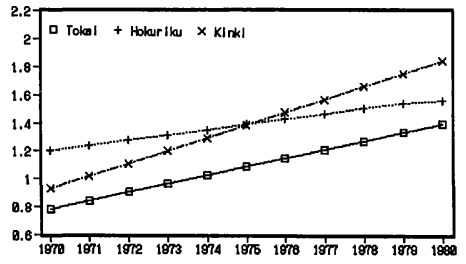

Fig. 25 Adaptive Regional Capital Coefficients (case 2)

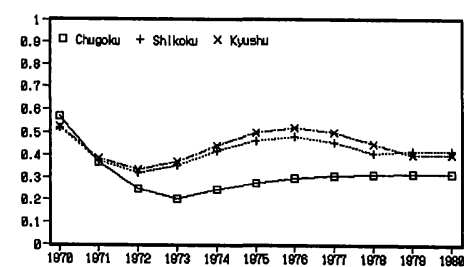

Fig. 23 Adaptive Regional Input Coefficients (case 2).

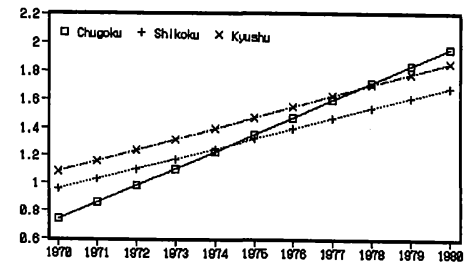

Fig. 26 Adaptive Regional Capital Coefficients (case 2). 
reference model

$$
X m(t+1)=f(X m(t), H)
$$

adaptive model

$$
X(t+1)=B^{-1}(t+1)(I-A m(t)-C S m \cdot V m(t)+B(t)) X(t)-B^{-1}(t+1) H
$$

adaptation laws

$$
\begin{aligned}
& C(t+1)=C(t)+(I+\Gamma(t))^{-1} K c \otimes \hat{\varepsilon}(t+1) X^{T}(t) \\
& D(t+1)=-B^{-1}(t+1)
\end{aligned}
$$

$B(t+1)$ is solved as follows;

$$
B(t+1)=(I-A m(t)-C S m \cdot V(t)+B(t)) C^{-1}(t+1)
$$

Setting up $k c_{i j}=10(j \neq 3), k c_{i 3}=3$, the degree of adaptation $D A(t)$ is shown in Fig. 27. Despite the fact that there is $0.21 \%$ error rate in $1971, D A(t)$ decreases uniformly after that period and approaches zero with $0.1 \%$ error rate in 1980 . From this result, the adaptive model is considered to trace the frame of NCNDP very well.

Now, Fig. $31 \sim 33$ show the adaptive variations of the regional capital coefficients which realize the regional outputs presented in Table 2. We observe in Fig. $31 \sim 33$ that every adaptive regional capital coefficient increases uniformly but is less than the actual values throughout this period. Specifically, Chugoku's and Tohoku's

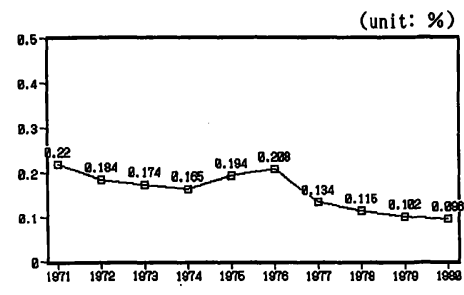

Fig. 27 Degree of Adaptation (case 3).

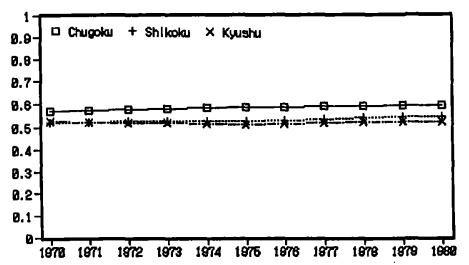

Fig. 30 Adaptive Regional Input Coefficients (case 3 ).

Fig. 29 Adaptive Regional Input Coefficients (case 3 ).

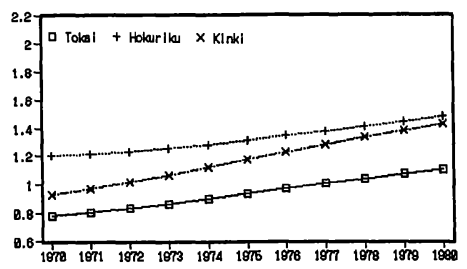

Fig. 32 Adaptive Regional Capital Coefficients (case 3 ).

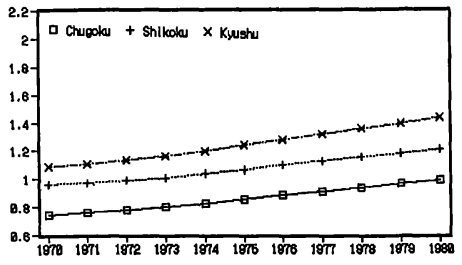

Fig. 33 Adaptive Regional Capital Coetncients (case 3 ).

adaptive capital coefficients are $50.7 \%$ and $63.5 \%$ of the actual one, respectively. These results show that an adaptation process with higher regional capital productivities is needed to achieve the goal of the NCNDP and whose growth rates are much higher than the 1970's actual values.

As mentioned above, a comparison of cases 1 and 2 with case 3 indicates that the adaptive interregional input coefficients vary excessively and those are unrealistic in actual sense. On the other hand, the adaptive interregional capital coefficients in case 3 change more slowly than the actual ones. Therefore case 3 is the most meaningful and rational among the three cases in the sense that it could result in realization of a high growth oriented and dispersed society in Japan, which was the goal aimed at by the NCNDP. In other words, this goal could be obtained by means of getting the regional productivities raised through effective utilization of regional resources, and strengthening the relationship between the local regions and the highly urbanized regions such as Kanto, Kinki, Tokai. 


\section{CONCLUSION}

This paper considered the processes of regional development embarked upon by the nine economic regions of Japan in the 1970's by using two approaches, that is, the regional turnpike and the Model Reference Adaptive Technique. Firstly, we found that the seven regions excluding Kanto and Chugoku developed along the regional turnpike for the most part of the duration under study. However, Kanto experienced a higher growth than the regional turnpike path, while Chugoku's actual output was less than the turnpike output.

Secondly, we regarded the frame of the New Comprehensive Nationwide Development Plan as a desirable development process, then considered how interregional structural changes would be needed to realize this frame. Our main finding is that under the assumption of the actual interregional input coefficients from 1970 to 1980 , the much higher regional capital productivities than the actual ones were needed in the interregional capital coefficients, and also the capital flow from the three major urbanized regions to the local regions should have been strengthened more further.

As stated above, the approaches which we adopted in this study have proved to be sound and feasible analytically and can be employed as a very effective and feasible method for the improvement of past development programs as well as in clarifying a future desirable regional structural changes, which has been studied in our previous papers.

\section{REFERENCES}

1) Miyata, Y. and Yamamura, E. : A study on the model reference adaptive I-O system, Chiikigaku Kenkyu, Vol. 17, pp. 1 31, Nov. 1987 (in Japanese).

2) Miyata, Y. and Yamamura, E. : A study on regional planning support system based on the theory of model reference adaptive system, Infrastructure Planning Review, Vol.6, pp.21 28, Dec. 1988 (in Japanese).

3) Yamamura, E. : Optimal and reference adaptive processes for the control of regional income disparities Papers of the Regional Science Association, Vol. 56, pp. 201 213, 1985.

4) Ministry of International Trade and Industry, Government of Japan : Interregional Input-Output Tables, Ookurasho Insatsukyoku, 1970, 1975, 1980, 1985 (in Japanese).

5) Economic Planning Agency, Government of Japan : Annual Report of Prefectural Accounts 1988, Ookurasho Insatsukyoku, 1988 (in Japanese).

6) Tsukui, J. : Application of a turnpike theorem to planning for efficient accumulation; An example for Japan, Econometrica, Vol. 36, No. 1, pp. 172 186, Jan. 1968.

7) Tsukui, J. and Murakami, Y. : Turnpike Optimality in Input-Output Systems; Theory and Application for Planning, North Holland, pp. 95 110, Jun. 1979.

8) Economic planning Agency, Government of Japan (ed.) : The Comprehensive Nationwide Development Plan, Ookurasho Insatsukyoku, 1962 (in Japanese).

9) Economic Planning Agency, Government of Japan (ed.) : The New Comprehensive Nationwide Development Plan, Ookurasho Insatsukyoku, 1969 (in Japanese). 